\section{Características epidemiológicas de la infección por VIH en Paraguay durante el año 2015}

\author{
Gloria Aguilar, Carlos Miguel Ríos-González, \\ Tania Samudio y Aníbal Kawabata
}

\section{Epidemiological characteristics of HIV infection in Paraguay during 2015}

To characterize the epidemiology of HIV in Paraguay, from january to december, in 2015. The epidemic remains focused in those groups under risk, predominantly male. The characteristics of the study population shows that most of the affected were from the central area and from the capital city, and sexual transmission remains the most common pathway, with delayed diagnosis, which is reflected by the percentage of people presenting a low CD4 cell count.

Key words: HIV, AIDS, epidemiology.

\section{Introducción}

$\mathrm{D}$ e acuerdo a los datos del Programa Conjunto de las Naciones Unidas sobre VIH/SIDA (ONUSIDA) se estima que 36,7 millones [34 millones-39,8 millones] de personas en todo el mundo padecían de la infección por VIH en el año 2015 y 2,1 millones [1,8 millones-2,4 millones] de personas contrajeron la infección durante este año. Mientras que en Latinoamérica se estima que hubo aproximadamente 100.000 [86.000-120.000] nuevas infecciones por $\mathrm{VIH}^{1}$.

De acuerdo a los datos el Programa Nacional de Control de VIH/SIDA/ ITS (PRONASIDA) del Ministerio de Salud Pública y Bienestar Social (MSPyBS) de Paraguay, la tasa anual de nuevos diagnósticos de infección por VIH presenta una tendencia ascendente desde el 2000 al 2013. Este hecho refleja el aumento del acceso al diagnóstico de la infección por VIH en la población de mayor riesgo, a través de la expansión de la consejería y del examen de VIH, a través de la consejería móvil y los Servicios de Salud $^{2}$. La investigación en este tema en nuestro país es baja ${ }^{3}$, por lo que se planteó como objetivo caracterizar la epidemiología de la infección por VIH en Paraguay, durante los meses de enero a diciembre de 2015.

\section{Material y Métodos}

Se realizó un estudio observacional, descriptivo de corte transversal, con muestreo no probabilístico por conveniencia sobre las características

\footnotetext{
Programa Nacional de Control de VIH/SIDA/ITS, Ministerio de Salud Pública y Bienestar Social, Paraguay (GA, TS, AK)

Facultad de Ciencias Médicas, Universidad Nacional de Caaguazú, Cnel. Oviedo, Paraguay (CMR-G).

Sin financiamiento.

Los autores declaran no tener conflicto de intereses.
}

Recibido: 19 de noviembre de 2016/ Aceptado: 9 de mayo de 2017

Correspondencia a:

Carlos Miguel Ríos González

carlosmigue_rios@live.com epidemiológicas de la infección por VIH en el Paraguay, durante los meses de enero a diciembre del año 2015.

La población de estudio la constituyeron las personas con diagnóstico de infección por VIH durante ese período. Fue considerado como diagnóstico para adultos y niños mayores de 18 meses, dos pruebas rápidas para VIH más el examen de Western Blot reactivo y en el caso de los niños menores de 18 meses, dos pruebas de carga viral mayores a 1.000 copias $/ \mathrm{ml}$.

\section{Resultados}

Ingresaron 1.430 pacientes con diagnóstico de infección por VIH durante los meses de enero a diciembre de 2015. La mediana de edad fue de 31 años (P25=24 P75=42). El 63,6\% (910) de los infectados fue de sexo masculino.

La distribución de acuerdo a los Departamentos, 38,8\% (521) fue del Departamento Central; 28,6\% (357) de Asunción y Alto Paraná 7\% (95).

Respecto a la población infectada por $\mathrm{VIH} ; 78,6 \%$ (1.124) fueron heterosexuales y $20,1 \%$ (288) eran hombres que tienen sexo con hombres (HSH).

El 30\% (423) fueron casos de SIDA y 6,4\% (92) fallecieron a causa de esta infección. La mediana del primer valor de linfocitos T CD4 fue de 241 céls $/ \mathrm{mL}(\mathrm{P} 25=93$ céls $/ \mathrm{mL}$ P75=412 céls $/ \mathrm{mL})$. En relación al valor de la primera determinación de la carga viral, la mediana fue de 928.793 céls $/ \mathrm{mL}$ (P25=9.833 céls $/ \mathrm{mL}$ P75=235.430 céls $/ \mathrm{mL})$.

\section{Discusión}

La tendencia mundial de la incidencia de la infección por VIH se estima normalmente por estudios de prevalencia en poblaciones centinela seleccionadas o, con menos frecuencia, en muestras representativas de la población.

La mediana de edad de nuestra serie fue de 30 años, lo cual difiere de otros estudios como Maartens y cols. ${ }^{4}$, donde fue de 25 años; sin embargo, se encuentra en el rango etario reportado por Ortblad y cols. en $2013^{5}$. Respecto a la distribución de género, la mayor frecuencia de nuevos diagnósticos se observó en hombres, semejante a lo descrito por Mansergh y cols. $^{6}$, en que consideran incluso al sexo masculino como factor de riesgo para la adquisición de la infección.

La prevalencia entre personas heterosexuales fue mayor en nuestra serie, lo cual difiere con datos reportados por los CDC en que es menor; sin embargo, han alertado que existe un incremento en esta población. Asimismo se ha visto un mayor número de casos de SIDA en heterosexuales ${ }^{7}$. En cuanto a la prevalencia en $\mathrm{HSH}$, se observaron proporciones similares a las series de Beyrer y cols. ${ }^{8}$ y Amirkhanian y cols. ${ }^{9}$, en especial en la población transexual ${ }^{10}$.

Como conclusión, en nuestra serie la epidemia sigue teniendo predominio en grupos de riesgo, de sexo masculino y en zonas urbanas. Es importante implementar políticas y estrategias para mejorar la captación de pacientes, y asimismo aumentar la promoción de la educación sexual en grupos de riesgo.

\section{Referencias bibliográficas}

1.- Programa Conjunto de las Naciones Unidas sobre el VIH/sida (ONUSIDA). Hoja informativa 2016. ONUSIDA; 2016. Disponible en: http://www. unaids.org/sites/default/files/media_asset/UNAIDS_FactSheet_es.pdf 
(Fecha de acceso: 10 de octubre de 2016).

2.- Objetivos de desarrollo del gobierno. Primer informe de gobierno. Gabinete Social. Presidencia de la República del Paraguay; 2010. Disponible en: http://www.dgeec.gov.py/parinfo/informacion/Resumen\%20Ejecutivo ODM\%202011.pdf (Fecha de acceso: 09 de octubre de 2016).

3.- Ríos-González C M. Análisis bibliométrico de la producción científica sobre VIH/sida en el Paraguay 1987-2015. SCientifica 2016; 14 (1): 49-49. Disponible en: http://www.revistasbolivianas.org.bo/scielo.php?script=sci arttext\&pid=S1813-00542016000100012\&lng=es. (Fecha de acceso: 10 de octubre de 2016).

4.- Maartens G, Celum C, Lewin S R. HIV infection: epidemiology, pathogenesis, treatment, and prevention. Lancet 2014; 384: 258-71.

5.- Ortblad K F, Lozano R, Murray C J. The burden of HIV: insights from the Global Burden of Disease Study 2010. AIDS 2013; 27: 2003-17.
6.- Mansergh G, Koblin B A, Sullivan P S. Challenges for HIV pre-exposure prophylaxis among men who have sex with men in the United States. PLoS Med 2012; 9 (8): e1001286.

7.- Centers for Disease Control and Prevention (CDC). HIV infection among heterosexuals at increased risk-United States, 2010. MMWR. Morb Mortal Wkly Rep 2013; 62: 183-8.

8.- Beyre C, Baral S D, van Griensven F, Goodreau S M, Chariyalertsak S, Wirtz A L, et al. Global epidemiology of HIV infection in men who have sex with men. Lancet 2012; 380: 367-77.

9.- Amirkhanian Y A. Social networks, sexual networks and HIV risk in men who have sex with men. Curr HIV/AIDS Rep 2014; 11: 81-92.

10.- Poteat T, Scheim A, Xavier J, Reisner S, Baral S. Global epidemiology of HIV infection and related syndemics affecting transgender people. J Acquir Immune Defic Syndr 2016; 72 Suppl 3: S210-9. 